\title{
Massive Particles for Brain Tractography
}

\author{
Ramón Aranda ${ }^{1}$, Mariano Rivera ${ }^{1}$, Alonso Ramírez-Manzanares ${ }^{2}$, Manzar \\ Ashtari $^{3}$ and James C. Gee ${ }^{4}$ \\ ${ }^{1}$ Centro De Invertigación en Matemáticas. \\ Guanajuato, Gto, México, 36240. \\ ${ }^{2}$ Universidad de Guanajuato, Departamento de Matemáticas. \\ Guanajuato, Gto, México, 36240. \\ ${ }^{3}$ Diffusion Tensor Imaging and Brain Morphometry Lab. Childrens Hospital of \\ Philadelphia. Philadelphia PA, USA, 19104. \\ ${ }^{4}$ Penn Image Computing and Science Laboratory (PICSL), Department of Radiology, \\ University of Pennsylvania. Philadelphia, PA, USA. \\ arac@cimat.mx,mrivera@cimat.mx, alram@cimat.mx, ashtari@email.chop.edu, \\ james.gee@uphs.upenn.edu
}

\begin{abstract}
We propose a method for estimating axonal fiber connectivity pathways (cerebral connectivity fibers) from Multi-Tensor Diffusion Magnetic Resonance Imaging (MTD-MRI). Our method uses multiple local orientation information provided by MTD-MRI for leading stochastic walks of particles. We perform stochastic walks on particles with mass which introduce inertia and gravitational forces that result in filtered trajectories. Afterwards, the fiber bunches are estimated with a clustering procedure based on terminal points that allows us to eliminate outliers. The method's performance is evaluated on MTD-MRI from realistic synthetic data, a diffusion phantom and demonstrated in real human brain data.
\end{abstract}

Key words: Tractography, MTD-MRI, Diffusion, Stochastic walks, Massive particles, Axonal fiber, Connectivity pathways, Tensor.

\section{Introduction}

White matter tractography is a procedure for estimating axonal fiber bundle in human brain to determine brain connectivity. Brain connectivity provides important information on normal human brain development and spectrum of neurological or neuropsychiatric disorders.

Axonal paths for in vivo brains can indirectly be estimated by measuring the molecular water diffusion at each point $x$ of the brain for a particular orientation; we denote such a diffusion by the funcion $\mathcal{D}_{x}: \mathbb{R}^{3} \times[0, p i)^{3}$. Water diffusion is the macroscopic effect of the molecular Brownian motion (microscopic motion) restricted by tissue walls. Then samples of $\mathcal{D}(x, \theta)$ can be obtained at each voxel, $x$, and for particular orientations, $\theta$, by means of Diffusion Weighted Magnetic Resonance Imaging (DW-MRI) modality. 
Tractography methods use the local diffusion orientations in order to estimate global connectivity information. The most popular parametric model for representing and analyzing DW-MRI is the named Diffusion-Tensor MRI (DTMRI). That technique fits a positive definite quadratic model to the logarithm of the DW-MRI signal. The measure of water diffusion in DT-MRI images can be computed from the Stejkal-Tanner [1] equation that relates only one tensor at $k$-th voxel, the inherent partial volume effect is not treated.

Given the evident difficulty of DT-MRI for managing more than one diffusion direction per voxel, there has been proposed more sophisticated parametric models; see for instance Refs. [2][3][4]. In particular, Multi-Tensor Diffusion MRI (MTD-MRI) can manage fibers that split, merge or cross [2]. For instance, Tuch et al. [5] proposed to use the Gaussian Mixture Model (GMM) (1). This model explains better the diffusion phenomenon for two or more fibers in a given voxel, as:

$$
S_{i, k}=S_{0, k} \sum_{j=1}^{J} \beta_{j} e^{\left(-b g_{i}^{T} T_{j, k} g_{i}\right)}
$$

where $S_{0, k}$ is the signal without diffusion at $k$-th voxel, $b$ is a constant acquisition parameter, $g_{i}$ is a unitary vector which $g_{i}=\left[g_{x_{i}}, g_{y_{i}}, g_{z_{i}}\right]$ indicates the direction on which is applied a independent magnetic gradient with $i=1,2, \ldots L$ and $L$ is the total number of applied gradients, $T_{j, k}$ is the $j$-th $3 \times 3$ tensor matrix symmetric positive definite, $S_{i, k}$ is the DW-MR measured signal by the application of $g_{i}, \beta_{j}$ is the contribution of tensor $T_{j, k}$ at the $k$-th voxel, with $\beta_{j} \epsilon[0,1]$ and $\sum_{j=1}^{J} \beta_{j}=1$, and finally $J$ indicates the total number of tensors within the voxel. The equation (1) shows the relationship between the signal without diffusion and the signal with diffusion on the direction $g_{i}$ at the $k$-th voxel. This equation is difficult to solve because the number of tensors need first be estimated and it is a highly non-linear system.

For solving (1), Ramírez-Manzanares et. al. [6] proposed a strategy for solving the inverse problem stated in (1). They relax the problem by using a fixed set of Diffusion Basis Functions (DBF) $\Phi=\left[\phi_{1}, \phi_{2}, \ldots, \phi_{N}\right]$. This set is not complete because is a discretization on a orientational 3D space. In this model $\phi_{j}$ is the diffusion weighted signal for the gradient vector $g_{i}$ and for the base (fixed) tensor $\bar{D}_{j}$. By using this model it is possible to solve (1) by solving an linear equation system with constrains. Next, for a tensor $T_{j, k}$ the $j$-th fiber orientation at the $k$-th voxel is estimated from the orientation of the first eigenvector, the one associated with the largest eigenvalue of $T_{j, k}$. This orientation is known as the Principal Diffusion Direction (PDD).

In this paper, we propose a novel method for estimating bundle axons fibers from MTD-MRI data. Our method is based on stochastic walks of massive particle, i.e. particles with mass. The particles' dynamic is governed by three components; a deterministic inertia force, a deterministic gravitational force (that results of the particles mass) and a stochastic medium selection. The stochastic components consists of the sample of the available orientations determined by the PDDs of the MTD model at each position. Multiple particles are seed at the position we want to analyze. Then, the particles move according the pro- 
posed dynamic. The stochastic path selection allows to explore multiple fibers meanwhile the inertia and gravity promotes smoothness and compacity.

Vectors with the particle temporal positions represent the particle trajectories or paths. Afterwards, bundle fibers are computed with hierarchical clustering method based on euclidean distances between terminal points of pairs of trajectories. We demonstrate our method by experiment with synthetic data, a realistic phantom and real in vivo brain human MT-MRI.

\section{Methods}

In the first part of this section, we introduce our procedure for computing stochastic walks of massive particles. We explain in detail how the next position for each particles is computer by taking into account the inertia, the gravitational influence of the surrounding particles and the medium (a multi-tensor field). The second part of this section explains our procedure for clustering random walks into fiber paths and to eliminate outliers.

\subsection{Stochastic Gravitational Tracktography.}

First, we introduce our notation. Let $N$ be the number of walking particles and $x_{t, m}$ be the position of the $m$-th particle at the iteration (step) $t$. Thus,

$$
x_{t+1, m}=x_{t, m}+\Delta d_{t+1, m}
$$

is the new position (at time $t+1$ ) of the $m$-th particle; where the motion direction is denoted by the unitary vector $d_{t+1, m}$ and $\Delta$ is a fixed step size. In our approach we compute this motion direction with the tree terms formula:

$$
d_{t+1, m}=\gamma_{1} d_{t+1, m}^{1}+\gamma_{2} d_{t+1, m}^{2}+\gamma_{3} d_{t+1, m}^{3}
$$

where the positive parameters $\gamma_{i}$, for $i=1,2,3$, weight the contributions of the directional vectors: $d^{1}, d^{2}$ and $d^{3}$, respectively. First direction, $d^{1}$, is associated with the medium where the particles are moving: it codifies the information of the local fiber orientation and is stochastically selected from the PDDs of the surrounding multi-tensor representation. Second direction, $d^{2}$, introduces an inertial component in the particle's trajectory. Last direction, $d^{3}$, is the resultant gravitational effect of the surrounding particles and promotes the particle to have a trajectory similar to the set. Before to explain the details for computing the motion direction of the terms in (3), we introduce the procedure for the particle initialization.

Particles Initialization. Given a seed pixel $\bar{x}, \beta^{*}=\arg \max _{\beta} \beta_{j}(\bar{x})$ is associated with most probable local orientation aligned with the unitary vector $v$, then the particles position are randomly initialized in the plane normal to $v$, with samples a two dimensional Gaussian centered at $\bar{x}$ and with variance $\sigma^{2}$ :

$$
\frac{1}{2 \pi \sigma^{2}} \exp \left(\frac{1}{2 \sigma^{2}}(x-\bar{x})^{T}\left(I-v v^{T}\right)^{-1}(x-\bar{x})\right) .
$$


Then, for all the particles, we set $d_{0,1: N}^{2}=v$ or $d_{0,1: N}^{2}=-v$, i.e. one direction of orientetion $v$.

Medium dependent direction $\left(\boldsymbol{d}^{\mathbf{1}}\right)$. The more important component of the particle dynamic is the effect of the medium on the selection of the possible paths, i.e. the local tissue structure. As we mentioned before, the computed tensors $T_{j}$ are associated with the local fiber paths and their respective mixture coefficients $\beta_{j}$, i.e. the proportion of the axonal fibers that are oriented with the PDD of each tensor. Also, we note that the particles positions have real valued coordinates (sub-pixel position). Thus, in general, a particle is in a neighborhood of eight pixels locations with integer valued coordinates where we have estimations of the multi-tensor model (1). Therefore, we introduce the hidden variables $y_{j}$ for $j=1, \ldots, 8$ that represent the positions of the eight neighbor voxels in the cube vertices containing $x_{t, m}$.

Thus, given the current position $x_{t, m}$ of the $m$-th particle at time (step) $t$ and its previous motion direction $d_{t, m}$. Then, by assuming independency, the probability density for the directions than the particle can follows has the form:

$$
P\left(d_{t+1, m}^{1}=\delta_{i, j} \mid d_{t, m}, x_{t, m}\right) \propto F_{\delta_{i, j}} F_{y_{i}}
$$

Then, the medium direction $d^{1}$ is randomly selected among the PDDs of the neighbor tensors taking into account the particle position and the current direction. Hence, the sampling of (5) is reduced to a random tournament. Now, we present the details of the conditional probability in (5) and the function that compute it.

First, let $\delta_{i, j}$ be the $j$-th PDD of $i$-th neighboring voxel $\left(y_{i}\right)$, then the probability weight being selected will depend on its likelihood with the previous motion direction and its size compartment, $\beta_{j, i}$ :

$$
F_{\delta_{i, j}}\left(\delta_{i, j}, \beta_{i, j}\right)=\beta_{j, i}\left|\delta_{i, j}^{T} d_{t, m}\right|^{w}
$$

where $w$ is a positive parameter that controls the variance function. Note that this function is maximum if the direction $d$ and $\delta_{i, j}$ are parallels, as it is illustrated by Figure 1(a).

On the other hand, each probability of selecting a PDD direction of a tensor in the neighborhood must be weighted by factors of how collinear $x_{t, m}$ and $y_{i}$ are with respect to direction $d_{t, m}$ and how close is $x_{t, m}$ from $y_{i}$. We write these weights in the second function, $F_{y_{i}}$,in $(5)$ as:

$$
F_{y_{i}}=F_{y_{i}}\left(y_{i}\right)^{1} F_{y_{i}}\left(y_{i}\right)^{2}
$$

where

$$
F_{y_{i}}\left(y_{i}\right)^{1}=\left(\frac{\left|d_{t, m}^{T}\left(x_{t, m}-y_{j}\right)\right|}{\left\|x_{t, m}-y_{i}\right\|}\right)^{z}
$$

weights the colinearity of previous step w.r.t. the orientation of neighbor $y_{i}$, where $z$ is a positive parameter that controls the variance function. A neighbor 


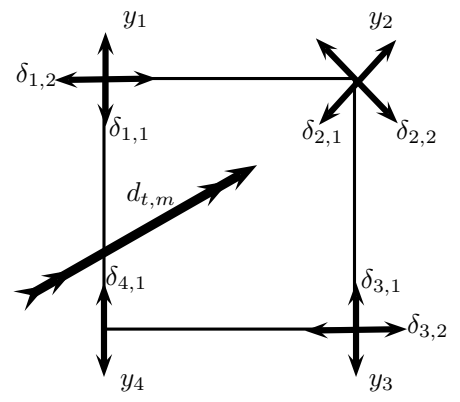

(a)

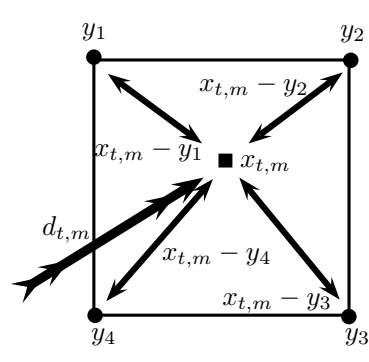

(b)

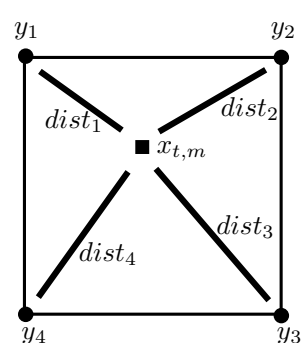

(c)

Fig. 1. Examples 2D of the function that form the probability of choosing a new PDD. (a) Factor of parallelism between $d_{t, m}$ with $d_{i, j}^{\prime} s$. (b) Factor of colinearity between $d_{t, m}$ with $\left(x_{t, m}-y_{i}\right)$. (c) Factor of closeness between $x_{t, m}$ and $y_{i}$.

voxel will have the largest value if it is aligned with the past trajectory, see Figure 1(b). Moreover,

$$
F_{y_{i}}\left(y_{i}\right)^{2}=\exp \left(-\frac{1}{2 \sigma^{2}}\left\|x_{t, m}-y_{i}\right\|^{2}\right)
$$

is the function that weights to neighbor voxel $y_{i}$ by a factor of proximity w.r.t. the current position. It depends on the Euclidean distance between the particle's position and neighboring voxel, as is illustrated by Figure 1(c).

Inertial force $\left(\boldsymbol{d}^{2}\right)$. Second direction, $d^{2}$, is exactly the previously used direction:

$$
d_{t+1, m}^{2}=d_{t, m}
$$

and it introduces an inertial component in the particle's trajectory.

Gravitational effect $\left(\boldsymbol{d}^{3}\right)$ The Newton's Universal Law of Gravitation states that the force exerted on a particle $x_{a}$ with mass $\omega_{a}$ by another similar $x_{b}$ with mass $\omega_{b}$ is inversely proportional to the square of the distance between them and directly proportional to product of their masses:

$$
F_{a b}=\bar{G} \omega_{a} \omega_{b} \frac{\hat{u}_{a b}}{\left\|x_{a}-x_{b}\right\|^{2}},
$$

where $\hat{u}_{a b}$ is the unit vector addressed from the particle $x_{a}$ to particle $x_{b}, \bar{G}$ the gravitational constant. Then, if we assume equal mass $\omega$ for the particles, then the gravitational force exerted between the particle $x_{t, m}$ and the rest of the particles is given by

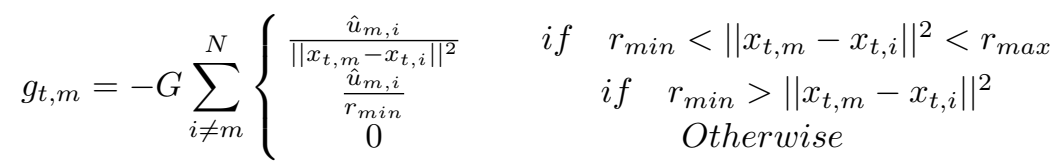


where $r_{\min }$ and $r_{\max }$ are the minimum and maximum distances respectively that one particle is from another one and $G=\bar{G} \omega^{2}$. We use this in order to keep the system stability: when two particles are too close the term $\left\|x_{t, m}-x_{t, i}\right\|^{2} \rightarrow 0$ thus $\frac{1}{\left\|x_{t, m}-x_{t, i}\right\|^{2}} \rightarrow \infty$, and vice versa when $\left\|x_{t, m}-x_{t, i}\right\|^{2}$ have a high value the gravitational force is close of zero. Thus, we use force (12) as the third component in our dynamic formula in (3), i.e

$$
d_{t+1, m}^{3} \equiv g_{t, m}
$$

Stop Criterion. We stop the walk of one particle if it reaches a voxel $k$ with Fractional Anisotropy (FA) of a DT-MRI lower than a given threshold $\tau \in(0,1)$. The $F A$ (Basser et al. [1]) on a voxel is defined by

$$
F A\left(D_{k}\right)=\sqrt{\frac{\left(\lambda_{1}-\lambda_{2}\right)^{2}+\left(\lambda_{1}-\lambda_{3}\right)^{2}+\left(\lambda_{2}-\lambda_{3}\right)^{2}}{2\left(\lambda_{1}^{2}+\lambda_{2}^{2}+\lambda_{3}^{2}\right)}}
$$

where $\lambda_{1} \geq \lambda_{2} \geq \lambda_{3}$ are the eigenvalues of the tensor $D_{k}$. Thus, our global Stop Criterion is reached when all the particle walks are stopped.

Summary. The algorithm 1 summarizes the random walks procedure.

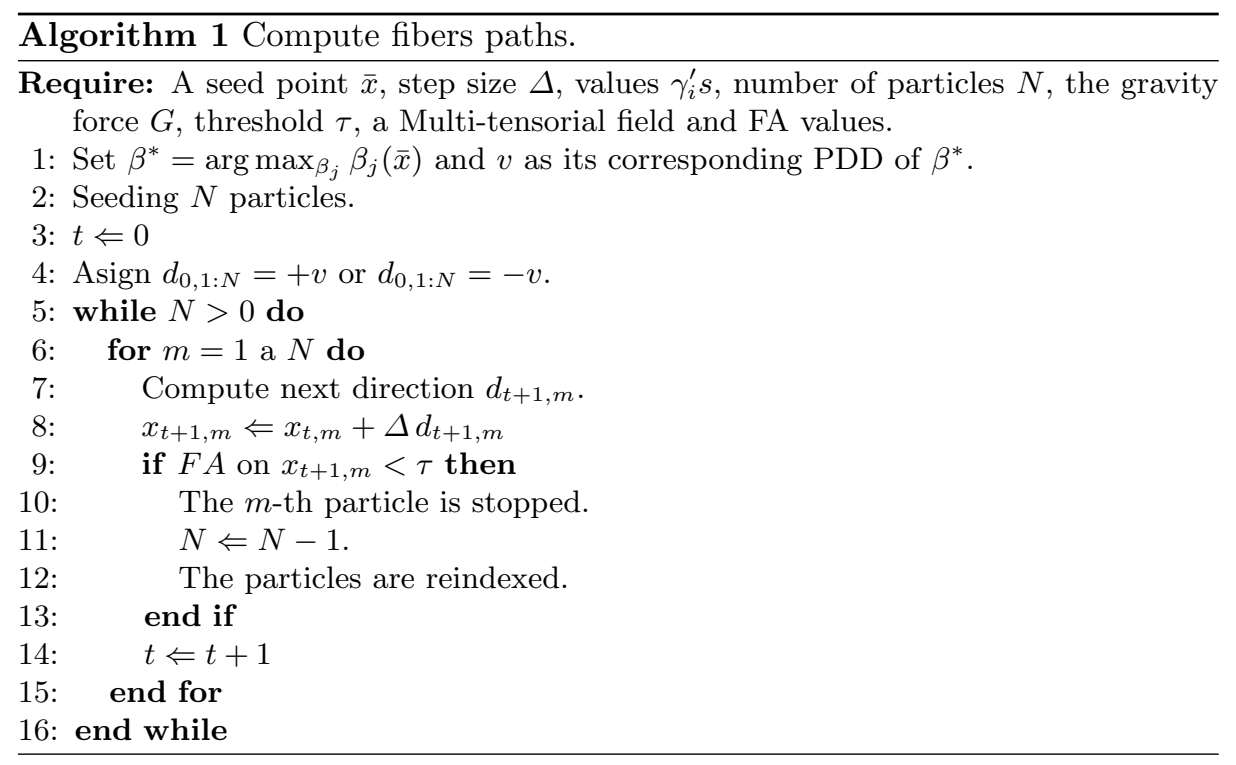

\subsection{Clustering and Outlier Rejection}

In order to estimate axonal fibers we need to cluster the fibers paths and discard the outliers paths. Note the high dimensionality of the data: one path have 
dimension from $\mathbb{R}^{3 \times M}$, assuming $M$ steps. Each path has an initial point, a trajectory and an ending point. According with our experiments, the most important feature for clustering particles paths are their final position (end-points). This can easy be understood from the cat that the initial particles points are fixed and as they spread as the number of iterations increases. Thus, we clasify the set of recovered pathways by means of a non parametric clustering method named hierarchical clustering. Hierarchical clustering algorithms usually are either agglomerative ("bottom-up") or divisive ("top-down"). For our clustering method, we use agglomerative algorithms, which starts with each element as a separate cluster and merge them into successively larger clusters[7]. We also use single linkage, also called nearest neighbor, as the method for compute the distance between clusters. Single linkage uses the smallest distance between objects in the two clusters, $c_{1}$ and $c_{2}$ as:

$$
d\left(c_{1}, c_{2}\right)=\min \left(\operatorname{dist}\left(\hat{x}_{c_{1}, i}, \hat{x}_{c_{2}, j}\right)\right), i \epsilon\left(1, \ldots, n_{c_{1}}\right), j \epsilon\left(1, \ldots, n_{c_{2}}\right)
$$

where $\hat{x}_{q, l}$ is an element $l$ of the cluster $q$ with $n_{q}$ elements and $\operatorname{dist}(a, b)$ is a distance measure (we use the Euclidean distance), in our case the $\hat{x}^{\prime} s$ are terminal points. Thus if $d\left(c_{1}, c_{2}\right)<c, c_{1}$ and $c_{2}$ are joined, where $c$ is a certain threshold of distance.

Once we compute the clusters, we discard the false clusters if they are composed by a percentage of pathways which is lower than a given percentage $\varphi$, namely, if the cluster contains few fibers it is eliminated.

Summary. The particle walks clustering is summarized in the Algorithm 2.

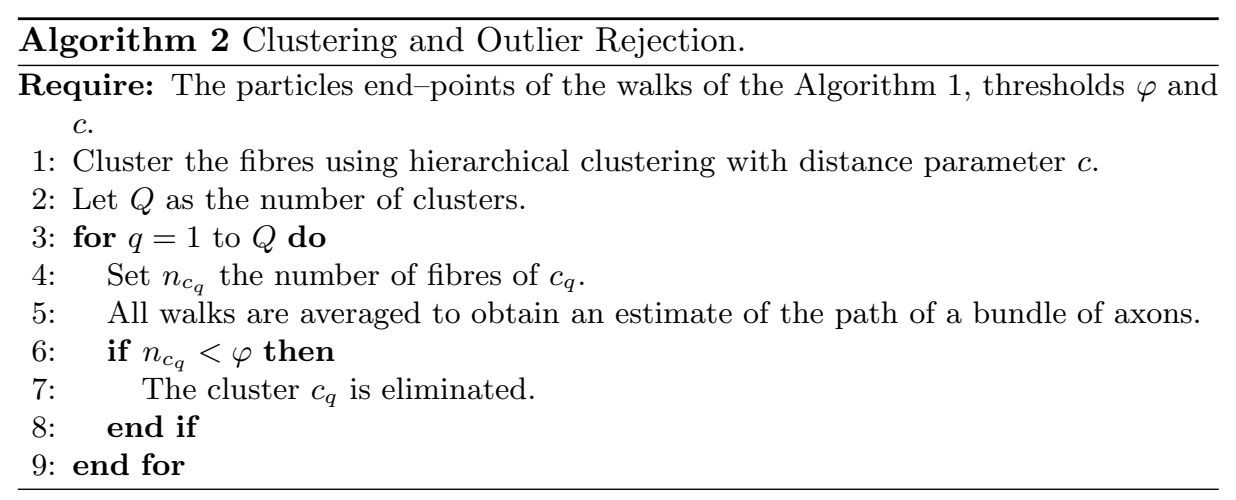

\section{Experiments and Results}

In order to show the performance of our method we use three different types of DW Data:

Synthetic data The DW-MRI signal was synthesized from the GMM (1). The DT principal eigenvalue was set to $1 \times 10^{-3} \mathrm{~mm}^{2} / \mathrm{s}$ and the second and 


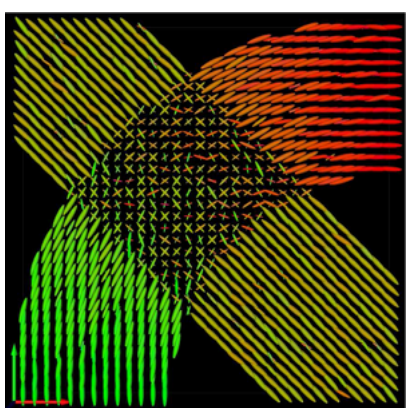

(a)

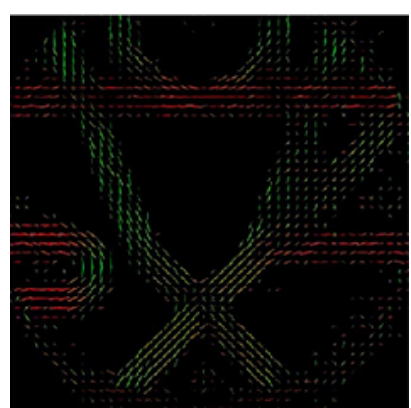

(b)

Fig. 2. Multi-Tensorial fields. (a) Synthetic data, (b) Diffusion Phantom data.

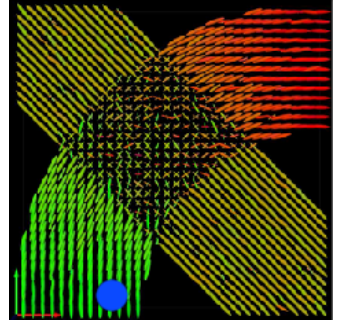

(a)

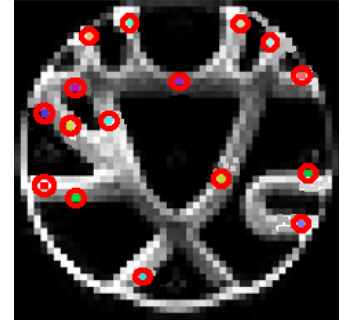

(b)

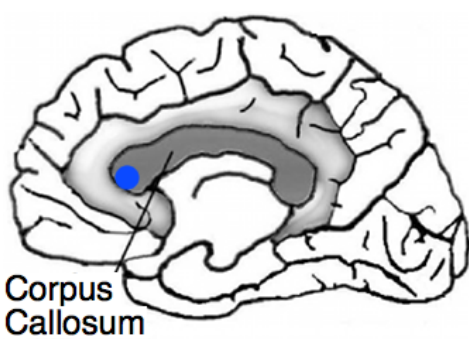

(c)

Fig. 3. Seed points of the differents data types. (a) Synthetic data: mark blue. (b) Diffusion Phantom data: red marks (c) Brain Human Data: blue mark and the corpus callosum.

third tensor eigenvalues were $2.22 \times 10^{-4} \mathrm{~mm}^{2} / \mathrm{s}, \mathrm{FA}=0.74$. The above values were taken from a sample of tensors observed in the brain data from a healthy volunteer. Rician noise was added to each measurement to produce $\mathrm{SNR}=9$. For these data, we have 1 repetitions. See Figure 2(a).

Data from a diffusion phantom. We used data acquired from a diffusion phantom [8]. Layers of hydrophobic acrylic fibres were interleaved and stack in each other to build fibre crossing configurations. Diffusion-weighted data were acquired on the $3 \mathrm{~T}$ Tim Trio MRI systems with 12-channel. The data is available at http://www.lnao.fr/spip.php?article112. For these data, we have only 2 repetitions. See Figure 2(b).

In vivo Brain Human Data. A single healthy volunteer was scanned on a Siemens Trio 3T scanner with12 channel coil. Acquisition parameters: singleshot echo-planar imaging, five images for $b=0 \mathrm{~s} / \mathrm{mm}, 64 \mathrm{DW}$ images with unique, isotropically distributed orientations $\left(b=1000 \mathrm{~s} / \mathrm{mm}^{2}\right), \mathrm{TR}=6700 \mathrm{~ms}, \mathrm{TE}=85$ $\mathrm{ms}, 90^{\circ}$ flip angle, voxel dimensions equal to $2 \times 2 \times 2 \mathrm{~mm}^{3}$. The approximated 


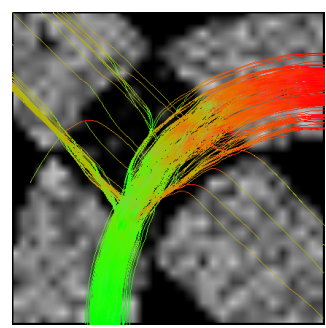

(a)

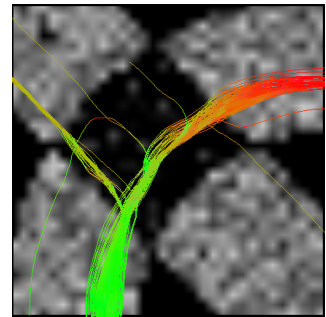

(d)

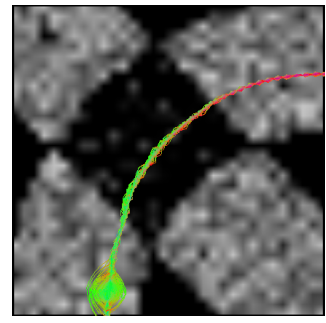

$(\mathrm{g})$

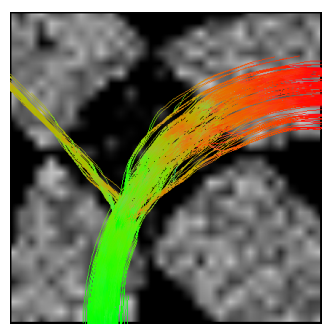

(b)

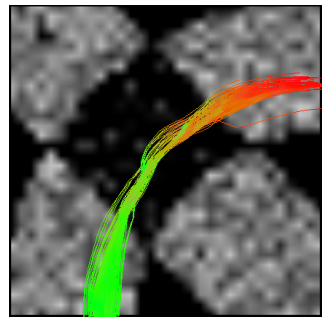

(e)

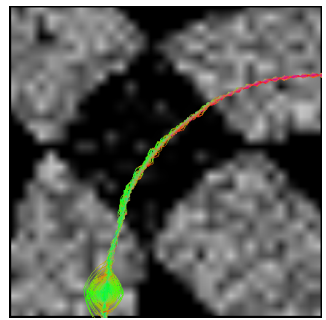

(h)

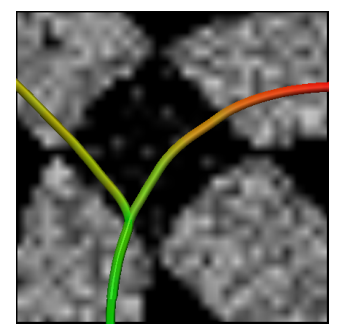

(c)

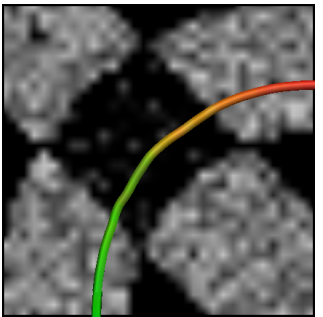

(f)

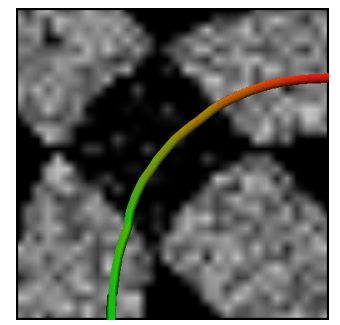

(i)

Fig. 4. Results step by step (by rows) of Traking fiber on synthetic data by using different values of $\mathrm{G}$. (a) $\mathrm{G}=0$, (d) $\mathrm{G}=0.0001$ and (g) $\mathrm{G}=0.002$. (b), (e) and (h) are the results without outliers, respectively. (c), (f) and (i) show the computed bundles.

Signal to Noise Ratio (SNR) is equal to 26 . For these data, we acquire 5 repetitions.

The first experiment was performed with synthetic data. The Figure 3 shows the results obtained, step by step, for the fibers crossing with different values of $\mathrm{G}$ and using as seed point the blue mark of image $3(\mathrm{a})$. We can see that when $\mathrm{G}=0$ is used many particles take a wrong way. On the other hand, when weak gravity is used, the above problem is corrected for the majority of particles but the method still explores the potential bifurcations.. Finally, when strong gravity is used the particles no longer can explore the medium because they remain together. This is not proper when there are bifurcations bacause the exploration is not allowed. 


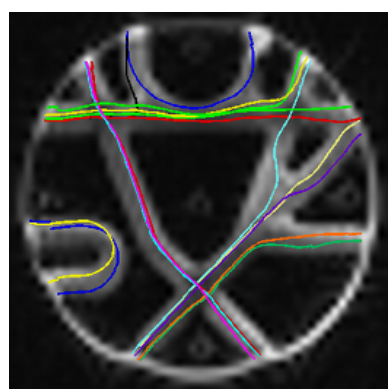

(a)

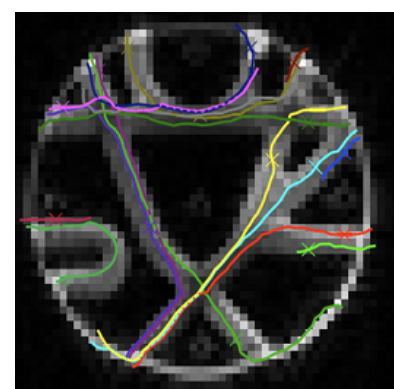

(b)

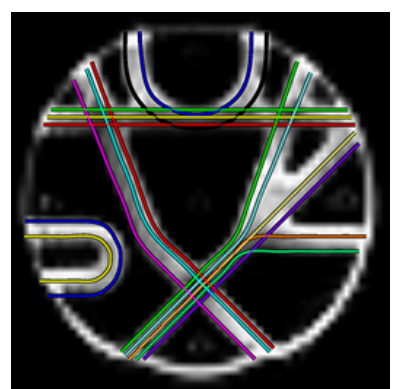

(c)

Fig. 5. Result of Traking fiber on diffusion phantom data: (a) our approach, (b) Method Ramírez-Manzanares et. al. and (c) ground truth.

Table 1. Root Mean Square Error using L2 norm between the obtained fibers with the method of Ramírez-Manzanares et. al. (RM) and ours (MP).

\begin{tabular}{ccccccccccccccccc}
\hline Fibers & 1 & 2 & 3 & 4 & 5 & 6 & 7 & 8 & 9 & 10 & 11 & 12 & 13 & 14 & 15 & 16 \\
\hline MP & 2.56 & 1.96 & 4.28 & 2.05 & 1.9 & 1.63 & 66.5 & 2.62 & 7.71 & 33.58 & 3.69 & 2.24 & 2.31 & 4.41 & 3.31 & 6.42 \\
RM & 4.98 & 39.65 & 53.84 & 6.02 & 7.36 & 2.65 & 71.1 & 14.77 & 10.2 & 9.41 & 21.51 & 14.83 & 20.01 & 2.94 & 16.37 & 14.35 \\
\hline
\end{tabular}

The second experiment was performed using the diffusion phantom data. The Figure 3 shows the visual comparison between the results obtained from the seed points in image 3(b) by using our approach, the method of RamírezManzanares et. al. [9] and the ground truth. Note that the visual comparation is representative since the diffusion phantom is basically in $2 \mathrm{D}$. In this manner, if a fiber is seen similar to ground truth, then, the error between the obtained fiber and the real fiber will be minimal. This can be seen also in Table 1. The Table 1 shows the numerical comparison, fiber by fiber, using L2 norm. Thus, one can see that we recover correctly 13 of 16 tracks. Instead, the method of RamírezManzanares et. al. recover correctly only 5 fibres. It is important to mention that all obtained tracks were performed with the same set of parameters, also, the presented fibers are the average from the group (cluster) with the highest number of walks.

The last experiment was performed using in vivo Brain Human data. The Figure 6 shows the obtained results using one seed point planted in the corpus callosum indicated by the blue mark in image 3(c). The Panel (a) shows the results without gavity force and the panel (b) is shown the tracks using $\mathrm{G}=0.0001$ and 500 particles. This shows very clear that when weak gravity is used the trajectories obtained are more consistent that when the gravity force is not used. On the other hand, the Figure 7 shows different views of the results of our approach for in vivo brain human data using 47 seed points around corpus callosum (see image $3(\mathrm{c})$ ), 1000 particles for each seed point and $\mathrm{G}=0.00005$. 


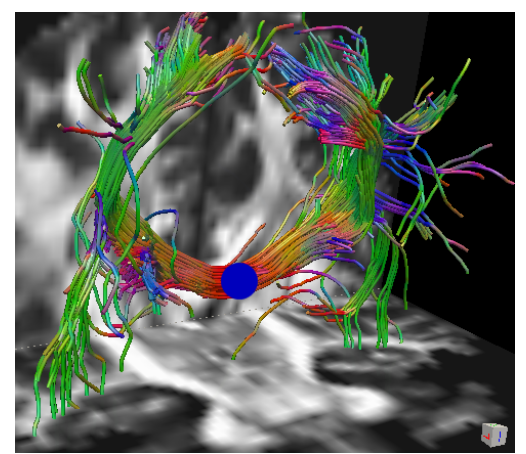

(a)

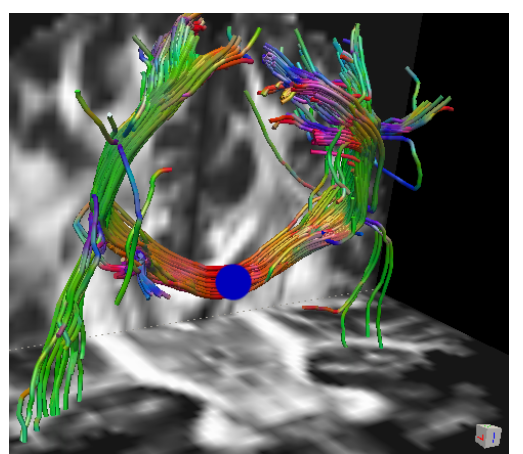

(b)

Fig. 6. Results of fiber tracking on in vivo brain human data using 500 particles and (a) $G=0$, (b) $G=0.0001$. The seed point is located on the blue mark in Figure 3(c).

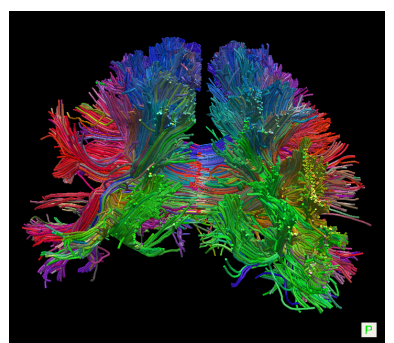

(a)

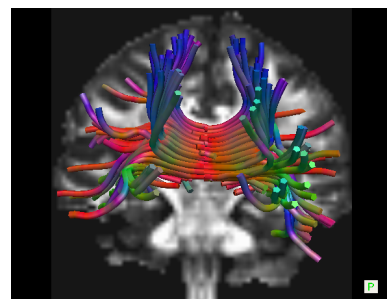

(d)

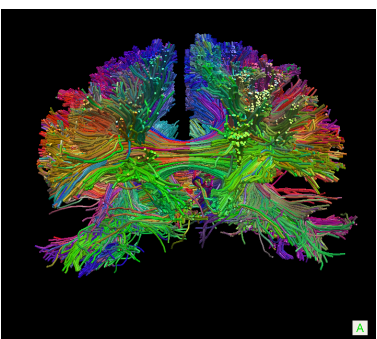

(b)

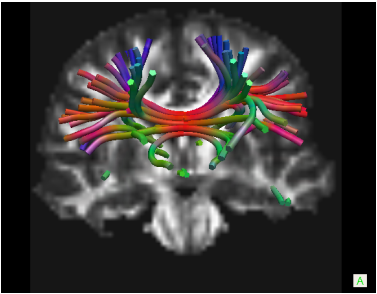

(e)

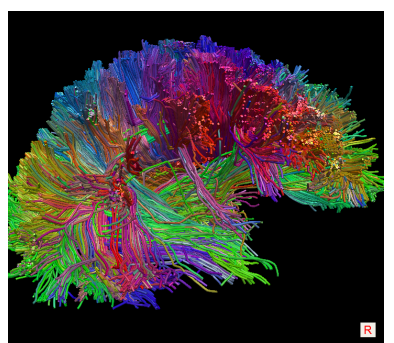

(c)

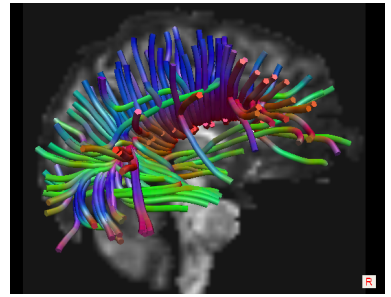

(f)

Fig. 7. Results of fiber tracking on in vivo human data using 47 seed points on corpus callosum, with $\mathrm{G}=0.00005$ and 1000 particles. (a), (b) and (c) Differents views of fibers without outliers, (d), (e) and (f) differents views of fiber Bundles.

These images show the estimated pathways without outliers. Also, one can see the averaged (main) connections in the panels (d), (e) y (f). 


\section{Conclusions}

This report presents a novel method for stochastic tractography based on massive particles. The particles dynamic depends on its previous direction (inertia), its current position, the orientation information of its neighbor voxels (medium), and on a new proposed gravitational term. The inertia, the medium and the gravitational force among particles promote smooth particle trajectories.

The gravitational force aids in the correction of particles trajectories as well as allowing medium exploration. According to our results, there is a compromise between the gravitational forces and particle spread in the medium (i.e. the capability of exploration). In practice, smaller values of gravity significantly improves the solution of w.r.t. the computed solutions without the gravity force.

In addition, we present a method for clustering particles comprising the pathway for the axonal fiber tracks. This clustering method allows removal of portion of the axonal bundles that have been wrongly estimated. True performance of the proposed method was demonstrated on synthatic and real human brain images.

Acknowledges. This work was supported by the Consejo Nacional de Ciencia y Tecnologia, Mexico, [MSc. Scholarship to R.A. and 61367-Y to M.R.].

\section{References}

1. Basser, P.J., Pierpaoli, C.: Microstructural and physiological features of tissues elucidated by quantitative-diffusion-tensor MRI. J. Magn. Reson. B 111 (1996)

2. Ramírez-Manzanares, A., Rivera, M.: Basis tensor decomposition for restoring intravoxel structure and stochastic walks for inferring brain connectivity in DT-MRI. Int. Journ. of Comp. Vis. 69 (2006) 77-92

3. Bergmann, O., Kindlmann, G., Peled, S., Westin, C.F.: Two-tensor fiber tractography. In: IEEE 2007 International Symposium on Biomedical Imaging (ISBI), Washington D.C., (2007)

4. Malcolm, J.G., Michailovich, O., Bouix, S., Westin, C.F., Shenton, M.E., Rathi, Y.: A filtered approach to neural tractography using the watson directional function. Medical Image Analysis 14 (2010) 58-69

5. Tuch, D.S., Reese, T.G., Wiegell, M.R., Makris, N., Belliveau, J.W., Wedeen, V.J.: High angular resolution diffusion imaging reveals intravoxel white matter fiber heterogeneity. Magn. Reson. Med. 48 (2002) 577-582

6. Ramírez-Manzanares, A., Rivera, M., Vemuri, B.C., Carney, P., Mareci, T.: Diffusion basis functions decomposition for estimating white matter intravoxel fiber geometry. IEEE Trans. Med. Imag. 26 (2007) 1091-1102

7. Tibshirani, T.H.R., Friedman, J. In: The Elements of Statistical Learning: Data Mining, Inference, and Prediction. Second Edition. Springer (2009)

8. Poupon, C., Kezele, B.R.I., Perrin, M., Poupon, F., Mangin, J.: New diffusion phantoms dedicated to the study and validation of high-angular-resolution diffusion imaging (HARDI) models. Magn Reson Med 60 (2008) 1276-1283

9. Ramírez-Manzanares, A., Rivera, M., Gee., J.C.: Depicting axon fibers on a diffusion phantom by means of hybrid DBF-DT data. In: Workshop Diffusion Modelling and Fiber Cup at MICCAI 2009., London, U.K. (2009) 1-4 